\title{
Recombinant Limosilactobacillus (Lactobacillus) delivering nanobodies against Clostridium perfringens NetB and alpha toxin confers potential protection from necrotic enteritis
}

\author{
Dharanesh Gangaiah $^{1}$, Valerie Ryan ${ }^{1}$, Daphne van Hoesel ${ }^{2}$, Shrinivas Mane ${ }^{1}$, Enid \\ McKinley $^{1}$, Nallakannu Lakshmanan ${ }^{1}$, Nandakumar Reddy ${ }^{1}$, Edward Dolk ${ }^{2}$, and Arvind \\ Kumar $^{1}$ \\ ${ }^{1}$ Elanco Animal Health Inc \\ ${ }^{2}$ QVQ Holding BV
}

February 16, 2022

\begin{abstract}
Necrotic enteritis (NE), caused by Clostridium perfringens, is an intestinal disease with devastating economic losses to the poultry industry. NE is a complex disease and predisposing factors that compromise gut integrity are required to facilitate $\mathrm{C}$. perfringens proliferation and toxin production. NE is also characterized by drastic shifts in gut microbiota; C. perfringens is negatively correlated with Lactobacilli. Vaccines are only partially effective against NE and antibiotics suffer from the concern of resistance development. These strategies address only some aspects of NE pathogenesis. Thus, there is an urgent need for alternative strategies that address multiple aspects of NE biology. Here, we developed Limosilactobacillus (Lactobacillus) reuteri vectors for in situ delivery of nanobodies against NetB and $\alpha$ toxin, two key toxins associated with NE pathophysiology. We generated nanobodies and showed that these nanobodies neutralize NetB and $\alpha$ toxin. We selected L. reuteri vector strains with intrinsic benefits and demonstrated that these strains inhibit C. perfringens and secrete over 130 metabolites, some of which play a key role in maintaining gut health. Recombinant L. reuteri strains efficiently secreted nanobodies and these nanobodies neutralized NetB. The recombinant strains were genetically and phenotypically stable over 480 generations and showed persistent colonization in chickens. A two-dose in ovo and drinking water administration of recombinant L. reuteri strains protected chickens from NE-associated mortality. These results provide proof-of-concept data for using L. reuteri as a live vector for delivery of nanobodies with broad applicability to other targets and highlight the potential synergistic effects of vector strains and nanobodies for addressing complex diseases such as NE.
\end{abstract}

\section{Hosted file}

MB032021120554.pdf available at https://authorea.com/users/460816/articles/556693recombinant-limosilactobacillus-lactobacillus-delivering-nanobodies-against-clostridiumperfringens-netb-and-alpha-toxin-confers-potential-protection-from-necrotic-enteritis 